374 (1960); ref. C. A. 55, 9531 h (1961). - 69. SQUIBB, R. L., Nature (London) 198, 317 (1963); 199, 1216 (1963). - 70. GERBER, G. B. und G. Gerber, Clin. chim. Acta (Amsterdam) 5, 607 (1960). - 71. Walz, D., A. R. Fahmy, G. Pataki, A. NiederWIESER und M. BRENNER, Experientia (Basel) 19, 213 (1963). 72. Keller, M. und G. Patakr, Helv. chim. Acta 46, 1687 (1963). - 73. Pataki, G. und M. Keller, diese Z. 1, 157 (1963). - 74. Drawert F., O. Bachmann und K. H. Rether, J. Chromatogr. (Amsterdam) 9, 376 (1962). - 75. PALMORK, K. H., Acta chem. scand. 17, 1456 (1963). - 76. Wallenfels, K. und A. Arens, Biochem. Z. 332, 217 (1960). - 77. Portugal, A. V., R. Green und T. M. Sutherland, J. Chromatogr. (Amsterdam) 12, 183 (1963). - 78. Lucas, F., L. T. B. Shaw und S. G. SMith, Analyt. Biochem. (New York) 6, 335 (1963). - 79. TongE, B. L., Nature (London) 195, 491 (1962). - 80. Pollara, B. und R.W. von KORFF, Biochim. biophysica Acta (Amsterdam) 39, 364 (1960). - 81. Russel, D. W., Biochem. J. 87, 1 (1963). - 82. SANGER, F., Biochem. J. 45, 562 (1949); BlaCkBourn, S., Nature (London) 163, 955 (1949); MrdDLEBrook, N., Nature (London) 164, 501 (1949); Green, F. CH. und L. M. KAY, Analytic. Chem. 24, 726 (1952); Schröder, W. A. und L. R. GonNen, J. Amer. chem. Soc. 75, 4615 (1953). - 83. Mrrls, G. L., Biochem. J. 50, 707 (1952); Braunitzer, G. und K. H. Reuther, Makromolekulare Chemie 18/19, 501 (1956). - 84. McFADDEN, M. L. und E. L. SMith, J. biol. Chemistry 214, 185 (1955); Partridge, S. M. und M. F. Davies, Biochem. J. 61, 21 (1955); Perrone, J. C., Nature (London) 167, 513 (1951). - 85. BeLl, P. H., Ann. New York Acad. Sci. 51, 897 (1949); Matheson, N. A., Biochem. J. 88, 151 (1963). - 86. SEKI, T. J., Biochemistry (Tokyo) 47, 253 (1960). 87. Partridge, S. M. und T. Swain, Nature (London) 166, 272 (1950). - 88. SteuerLe, H. und E. Hirle, Biochem. Z. 331, 220
(1959); Hille, E., Biochem. Z. 333, 269 (1960); HörmanN, H. und H. von Portatius, Hoppe-Seyler's Z. physiol. Chem. 315, 141 (1959). - 89. Wooley, D. W., J. biol. Chemistry 179, 593 (1949). - 90. Lieboldt, B. und G. Braunitzer, Hoppe-Seyler's Z. physiol. Chem. 316, 170 (1959); 317, 287 (1959). - T91. LowTHWER, A. G., Nature (London) 167, 767 (1951). - 92. Mrller, G. L., Nature (London) 165, 403 (1950). - 93. Biserte, G., J. W. HoLlemanN, J. Hollemann-Dehove und P. Salutiere, Chromatogr. Rew. (Amsterdam) 2, 59 (1960). - 94. Biserte, G. und R. Osteaux, Bull. Soc. Chim. biol. 33, 126 (1951). - 95. Levy, A. L., Nature (London) 174, 126 (1954). - 96. Braunitzer, G., Chem. Ber. 88, 2025 (1955); Phillips, D. M. P., Biochem. J. 68, 35 (1958). - 97. MeLLoN, E. F., H. K. Korn und S. R. Hoover, J. Amer. chem. Soc. 75, 1675 (1953). - 98. Redfield; R. R. und C. B. Anfinsen, J. biol. Chemistry 221, 385 (1956). - 99. BrenNer, M., A. Niederwieser und G. Pataki, Experientia (Basel) 17, 145 (1961). - 100. BrenNer, M., A. Nieder wreser und G. Pataki, Seite $426 \mathrm{ff}$. in Zitat 36. - 101. Patakr, G. unveröffentlicht; Patakr, G. und M. KelLER, Helv. chim. Acta 47, 787 (1964). - 102. WADA, T., Dai 2kai Nippon Isotope Kaigi Hobunshu 2, 513 (1958); ref. C. A. 55, 7531 (1961). - 103. Beale, D. und J. K. Whitehead, Tritium Physical Biological Sci. (Vienna) 1, 179 (1962). - 104. PatakI, G., Chimia (Aarau, Schweiz) 18, 23 und 24 (1964). - 105. MuNNIER, J., J. Chromatogr. (Amsterdam) 1, 524 (1958). - 106. Sanger, F. und E. O. P. Thompson, Biochim. biophysica Acta (Amsterdam) 71, 468 (1963). - 107. Edwards, C. H., E. L. Gradston, L. B. Carter und G. Edwards, J. Chromatogr. (Amsterdam) 2, 188 (1959). - 108. Patakı, G., Dünnschichtchromatographie in der Aminosäure- und Peptidchemie, Walter de Gruyter \& Co. Berlin, in Vorbereitung.

Dr. phil. G. Pataki, Ing. Chem.

Bașel (Șchweiz), Schanzenstr. 46

\title{
Isolierung und Bestimmung des Mesobilifuscins
}

\author{
Von \\ Miroš MORAVEC \\ Aus dem Pyrrol-Laboratorium der Klinik für Virus-Hepatitis in Prag-Motol (Leiter: Prim. Dr. med. O. Soušse)
}

(Der Schriftleitung zugegangen am 22. Januar 1964)

\begin{abstract}
Es wird über die chemischen und physikalisch-chemischen Eigenschaften des im Organismus nativ vorkommenden polymerisierten Dipyrrols Mesobilifuscins berichtet. Die auf Grund dieser Eigenschaften ausgearbeiteten Isolierungsverfahren sind im Einzelnen angeführt und analysiert. Die von uns angeführten Bestimmungsmethoden im Stuhl, in der Galle und im Harn erzielen eine große Reinheit des Präparates. Die Meßempfindlichkeit liegt im Bereiche von Milligrammprozent. Die Verfolgung des Mesobilifuscins im menșchlichen Körper wird in der Zukunft sowohl zur weiteren Klärung der Fragen des Pyrrolaustausches, als auch zur besseren Einsicht in die Probleme der Leber- und Blutpathologie beitragen.
\end{abstract}

The chemical and physical-chemical properties of the polymerised dipyrrole, mesobilifuscin, which occurs naturally, are reported. The isolation procedures, based on these properties, are described in detail. Our methods of analysis for faeces, bile and urine give a very pure preparation. The determination is sensitive to increments of $1 \mathrm{mg}$. \%. Measurements of mesobilifuscin in humans will contribute to our knowledge of pyrrole exchange and to the problems of liver and blood pathology.

Bei der Erforschung der Gallenfarbstoffe wird in der letzten Zeit den Dipyrrolfarbstoffen, welche im Organismus in erster Linie durch das Mesobilifuscin
(„MBF“) vertreten sind, eine erhöhte Aufmerksamkeit gewidmet (1-6). Während jedoch bei den Tetrapyrrolverbindungen die Hauptprobleme ihrer Chemie, 
Physiologie und Pathophysiologie enträtselt sind oder gelöst werden, sind bei den Dipyrrolsubstanzen, welche für den Hämstoffwechsel und für die Leberpathologie von nicht minderer Bedeutung sind, die Grundfragen erst in groben Umrissen geklärt oder noch vollständig dunkel $(2,7,8)$. Die Hauptursache liegt dabei nicht in der Unterschätzung ihrer Bedeutung, sondern in der prinzipiellen Schwierigkeit ihrer Erfassung wegen der zeitraubenden und technisch anspruchsvollen Identifizierungs- und Isolierungsmethoden $(2,7)$. Die Eigenschaften der Dipyrrole und des MBF sind von ihrem physikalisch-chemischen Zustand abgeleitet (8). Das $\mathrm{MBF}$ ist nämlich ein Gemenge von verschiedenartigen vielfaltigen Polymeren der 2 isomeren Dipyrrole (8). Mithin gehört das MBF vom chemischen Standpunkt zur Makromolekularchemie, deren Lehrsätze beim Arbeiten mit dieser Dipyrrolsubstanz zu berücksichtigen sind. Maßgebend ist dabei in erster Linie ihre hohe Adsorptionskraft zur festen Phase bei der Anwendung der Adsorptionschromatographie: die Adsorption des $\mathrm{MBF}$ an das Aluminiumoxyd ist im Vergleich mit anderen Pyrrolfarbstoffen erheblich und das MBF geht sehr schwer in die flüssige Phase im Vergleich mit den monomeren Pyrrolfarbstoffen. Wichtig ist weiter die verschiedene Löslichkeit des MBF in einer Reihe von polaren, weniger polaren und unpolaren Lösungsmitteln (Alkohol, Wasser, Chloroform), die vom Polymerisationsgrad abhängig ist $(9,10)$. In konzentrierten Säuren, z. B. Essigsäure sind die räumlich angeordneten hochgradigen Polymere löslich (10). Nach SIEDEL ist das MBF in Essigsäure, ihren Estern, Alkohol, Pyridin und Chloroform leicht löslich, in Äther, Petroläther, Ligroin und Aceton fast unlöslich $(7,8)$. SIEDEL unterscheidet 5 Gruppen des MBF nach ihrer Löslichkeit (8):

\section{Wasserlösliche}

II. Wasserunlösliche, wobei diese wieder getrennt werden können in

1. chloroformlösliche

2. methanollösliche

3. amylalkobollösliche

4. unlösliche.

Tab. 1

Löslichkeit des Mesobilifuscins (Werte in \%)

\begin{tabular}{ccccc}
\hline $\begin{array}{c}\text { Lösungs- } \\
\text { mittel }\end{array}$ & $\begin{array}{c}\text { Löslichkeit } \\
\text { des FMBF } \\
\text { (SIEDEL) }\end{array}$ & $\begin{array}{c}\text { Erweiterte } \\
\text { Löslichkeit }\end{array}$ & $\begin{array}{c}\text { Löslichkeit } \\
\text { des UMBF }\end{array}$ & $\begin{array}{c}\text { Polymeri- } \\
\text { sationsgrad }\end{array}$ \\
\hline
\end{tabular}

\begin{tabular}{|c|c|c|c|c|}
\hline $\mathrm{CH}_{3} \mathrm{COOH}$ & & 28 & 50 & \\
\hline $\mathrm{CHCl}_{3}$ & 5 & $3,5$ (auch in $B)$ & & \\
\hline $\begin{array}{l}\mathrm{C}_{5} \mathrm{H}_{8} \mathrm{OH} \\
\mathrm{C}_{4} \mathrm{H}_{6} \mathrm{OH}\end{array}$ & 5 & $\begin{array}{c}6(\text { auch in B) } \\
6,5\end{array}$ & & \\
\hline $\mathrm{CH}_{3} \mathrm{OH}$ & 45 & $\begin{array}{c}28 \text { (Teil in E, } \\
\text { Teil in B) }\end{array}$ & 50 & $\begin{array}{c}\downarrow \\
\text { abnehmende }\end{array}$ \\
\hline $\begin{array}{l}\mathrm{H}_{2} \mathrm{O} \\
\text { unlöslich }\end{array}$ & $\begin{array}{c}45 \\
\text { anwesend }\end{array}$ & $\begin{array}{l}28 \text { (auch in E) } \\
\text { anwesend }\end{array}$ & abwesend & $\begin{array}{c}\text { Poly- } \\
\text { metisation }\end{array}$ \\
\hline
\end{tabular}

Ableirzungen: FMBF: Faeces-Mesobilifuscin; UMBF: Urin-Mesobilifuscin; B: Butanol; E: Essigsäure.
Diese Einteilung des MBF nach der Löslichkeit weist auf verschiedene Grade der Polymerisation hin. Nach den Maximen der Makromolekularchemie sind hochgradig polymerisierte Stoffe in weniger polaren Solventien löslich $(9,10)$. Da der Prozentanteil der einzelnen Löslichkeitsfraktionen bei unseren Analysen immer der gleiche war - unabhängig von der Reihenfolge der verwendeten Solventien - glauben wir, daß bloß MBF von einem bestimmten Polymerisationsgrad oder kleine Gruppen von einigen Polymerisationsstufen in die einzelnen Lösungsmittel aufgenommen werden.

Bei der eigenen Arbeit, die auf die Isolierung enteraler Fuscine abzielte, entdeckten wir außer den von SIEDEL genannten Gruppen auch noch Fuscine, die im Butylalkohol und in Essigsäure löslich waren; diese wurden nach vorheriger Extraktion der klassischen Fraktionen gewonnen, so daß die amylalkohollösliche, chloroformlösliche und ein Teil der methanollöslichen Fraktion sich als butanollöslich (höhere Polymere?), die wasserlösliche und ein Teil der methanollöslichen Fraktion sich als in Essigsäure löslich erwiesen (Tab. 1). Die Unabhängigkeit der Reihenfolge der Lösungsmittel hat somit für Butanol und Essigsäure keine Geltung. Es scheint, daß die Verfolgung der einzelnen MBF-Fraktionen nach ihrer Löslichkeit (nach ihrem Polymerisationsgrad) klinisch von Bedeutung sein wird. - Unter anderen physikalischen Eigenschaften des MBF sollen noch der Schmelzpunkt (169-170 $)$ und die hohe Hygroskopizität erwähnt werden. Obzwar einzelne nicht polymerisierte Dipyrrole bereits in Kristallform erhalten wurden, konnte das MBF nicht zur Kristallisation gebracht werden. Es ist wahrscheinlich, daß das MBF zu den nichtkristallisierenden Makromolekülen gehört. In konzentrierten Säuren, z. B. Salzsäure (SIEDEL) wird, besonders beim Sieden, der Polymerisationsgrad erhöht. Nach eigenen Erfahrungen wächst das Molekül des MBF auch nach längerem Aufbewahren in konzentrierter Essigsäure.

Ein großes Hindernis bei der Verfolgung der Mesobilifuscine besteht darin, daß keine empfindliche, chemische Farbreaktion $\mathrm{zu}$ ihrer Identifizierung vorliegt. Mesobilifuscine zeigen keine der bekannten Gallenfarbstoffreaktionen, weder mit Gmelins Reagens, noch mit Dimethylaminobezaldehyd oder Diazobenzolsulfonsäure. Auch die Pentdyopentreaktion ist negativ. Mit Zinkacetat bilden Fuscine ein schwer lösliches, nicht fluoreszierendes Komplexsalz. Reines MBF zeigt ein uncharakteristisches Absorptionsspektrum mit einem breiten Maximum zwischen 400 und $450 \mathrm{~m} \mu$ und mit einem langsamen Abfall in höheren Wellenlängen zum Nullpunkt (7). Die Identifizierung des MBF gelingt somit ausschließlich durch eine zeitlich und materiell ziemlich anspruchsvolle Isolierung, eine Methode, die wenig empfindlich ist. Auch wird die Ausbeute durch gewisse Präparationsverluste ziemlich vermindert. Wir sind überzeugt, daß sich dadurch viele Mesobilifuscinurien, einschließlich der möglichen physiologischen Mesobilifuscinurie, dem Nachweis entziehen und daß die quantitative Mesobilifuscinbestimmung erheblich unterschätzt wird. 


\section{Isolierung des MBF aus dem Stuhl}

W. SIEdEL gewann im Jahre 1939 das MBF aus Bilirubin (7). In demselben Jahr gelang diesem Autor die erste Isolierung des Myobilins, das später mit dem MBF identifiziert wurde, aus dem Stuhl des Myopathikers. Die von SIEDEL angegebene Methode war folgende (7):

Stuhl ungetrocknet bei Zimmertemperatur zweimal 6 Stdn. mit Methanol ausgezogen; Extrakt eingeengt und Rückstand mit Chloroform aufgenommen; Chloroformlösung mit Wasser gewaschen, durch Filtrieren getrocknet und eine kleine Menge der Chloroformlösung mit mehrfacher Menge von Petroläther versetzt. Der ausgeflockte Niederschlag in Methanol gelöst und durch $\mathrm{HCl}$ verestert. Der Alkohol im Wasserbad vertrieben, der Rückstand nochmals mit Chloroform aufgenommen, mit Wasser gewaschen und mit Petroläther ausgefällt. Niederschlag erneut in Chloroform gelöst und chromatographisch gereinigt; die Chloroformlösung wurde auf eine mit Äther getränkte AluminiumoxydSäule (4 cm Durchmesser, $30 \mathrm{~cm}$ Höhe) aufgegossen und zunächst mit einer Mischung von Chloroform-Âther $1: 10$ entwickelt. Dabei wurde ein gelber, eiweißhaltiger Teil ausgewaschen, worauf mit Chloroform- ̈̈ther-Gemisch 1:2 weiter entwickelt wurde. Hierbei wurde eine unter der obersten braunen MBFMethylester-Zone befindliche, mehr gelbliche Schicht, die Urobilin- und Sterkobilinmethylester enthält, ausgewaschen.

\section{W. SIEDEL hat diese Methode später abgeändert (8):}

\section{Neuere Darstellung von MBF aus Faeces nach} SIEDEL

Faeces, im Exsiktor über $\mathrm{P}_{2} \mathrm{O}_{5}$ getrocknet, werden pulverisiert und auf dem Wasserbad unter Rückfluß mit Methanol-Salzsäure 1 Std. erhitzt. Die Lösung färbt sich dabei dunkel-rot braun. Durch wiederholtes Filtrieren, erst durch Watte, dann auf der Nutsche durch Filtrierpapier wird eine klare Lösung erhalten. Der Rückstand ist nach dem Waschen mit Methanol graugefärbt, ein Zeichen, daß der gesamte braune Farbstoff entfernt ist. Nun wird das Filtrat, nachdem es mit Wasser versetzt worden ist, erschöpfend mit Chloroform extrahiert. Die vereinigten Chloroformauszüge werden dann mit $2 n$ Natriumcarbonatlösung gewvaschen, durch Filtrieren getrocknet und auf ein kleines Volumen eingeengt. Schließlich wird das restliche Chloroform mit Methanol unter Sieden verdrängt. Hierauf wird der in der methanolischen Lösung vorliegende rohe MBF-methylester nach Zugabe von Natriumhydroxyd in der Hitze verseift. Nach Neutralisation der alkalischen Lösung mit $2 n$ Schwefelsäure wird das MBF durch Ausschütteln mit Chloroform in dieses übergeführt. Die Chloroformlösung wird nunmehr auf ein kleines Volumen eingeengt und mit Petroläther (Ligroin) versetzt. Das MBF fällt dabei in Flocken aus, während eventuell vorhandene Fettsäuren in Lösung bleiben. Die Flocken werden abfiltriert und erneut mit Methanol- $\mathrm{HCl}$ verestert. Der Ester wird wieder wie oben in Chloroform übergeführt. Nachdem diese Chloroformlösung mit Natriumcarbonatlösung und Wasser neutral gewaschen ist, wird sie auf eine mit Äther getränkte Säule von Aluminiumoxyd aufgetropft. Die Entwicklung des Chromatogramms geschieht mit einem ChloroformÄthergemisch 1:3. Von den hierbei ausgebildeten Zonen wird die oberste, die den MBF-methylester enthält, isoliert und in der Hitze mit Eisessig behandelt. Beim Einengen der abgetrennten dunkelbraunen Eisessiglösung im Vakuum hinterbleibt der MBFmethylester. Er kann wieder in Alkohol oder Chloroform gelöst werden. Durch Verseifung mit Natriumhydroxyd wie oben und Fällung mit $2 n$ Schwefelsäure wird das MBF erhalten. Es stellt ein Gemisch mehrerer Assoziationsgrade, bzw. polymerer Stufen dar.

W. SIEDEL bestimmte auch die chemische Formel des MBF und stellte seine Tendenz zur Polymerisation und alle seine physikalisch-chemischen, oben angeführten Eigenschaften fest. - Zwecks weiterer Verfeinerung der Forschung und Bestimmung der mengenmäßigen Grundlage war es notwendig, eine Methode zur quantitativen Analyse des MBF auszuarbeiten. Es handelte sich sowohl um die maximale Ausbeute des MBF aus dem biologischen Material, als auch um die Art der Nachweismethode des gewonnenen reinen MBF. Die von A. Sigrid Gilbertsen, P. Lowry, Violet HawkINSON und C. J. WATSON im Jahre 1959 ausgearbeitete Methode erfüllt alle diese Erfordernisse (2).

\section{Quantitative Bestimmung des MBF nach Gilbertsen und Mitarbeitern (1959)}

Der wässerige Stuhlauszug wird bei $\mathrm{pH}=4,0$ mit Petroläther versetzt, um von Stercobilinogen befreit zu werden. Der wässerige Rückstand wird auf $80^{\circ}$ erwärmt und über die Nacht -stehen gelassen (Chromogen verwandelt sich in das Pigment). Am nächsten Tag wird die wässerige Lösung dreimal nacheinander mit 2-proz. Essigsäure-Butanol ausgezogen, wobei für die Extraktion etwa $75 \mathrm{~m} l$ Butanol verwendet werden. Butanol wird nachher dreimal mit Wasser ausgewaschen und filtriert. Der Rückstand wird bei Unterdruck auf weniger als $5 \mathrm{~m} l$ eingeengt. Dann werden etwa $30 \mathrm{~m} l$ Methanol und $10 \mathrm{ml}$ der gesättigten Zinkacetatlösung in Methanol zugesetż. Die Lösung wird für gewisse Zeit stehen gelassen, um die Bildung vom Zinkkomplex des Dipyrrylmethens zu ermöglichen. Der Niederschlag wird durch Filtration abgetrennt und mit Methanol gewaschen; nachher wird es mit dem Gemisch von Methanol-Schwefelsäure 20:1 vetestert. Der veresterte Zinkkomplex̃ wird dann nach 24 Stunden mit Chloroform extrahiert und weiter mit Wasser, 3-proz. Ammoniumhydroxyd und 7-proz. Natriumchlorid gewaschen. Demnächst wird der Dipyrrylmethenkomplex auf Umkehrphasen einer mit Äther gesättigten Aluminiumoxydsäule gereinigt. Die Säule wird zuerst mit dem Gemisch von Chloroform-Äthet 1:10, dann mit Chloroform- $\AA$ ther $1: 3$ und schließlich mit reinem Chloroform gewaschen. Zuletzt wird die oberste braune Schicht mit siedender Essigsäure ausgewaschen und nach wiederholtem Waschen mit Äther gefüllt. Zur quantitativen Messung wurde die optische Dichte am,Evelyn“Kolorimeter unter Benützung vom Filter $490 \mathrm{~m} \mu$ bestimmt. Die Vergleichlösung wurde durch die Verarbeitung von Mesobilirubinogen, das - nach SIEDEL und MöLLER - mit Bleitetraacetat oxydiert wurde, hergestellt. In dem auf diese Weise gewonnenen MBF schwankte der prozentuelle Stickstoffanteil zwischen 4 und 5\% (theoretische Stickstoffmenge: 8,8\%) (13).

Um eine noch größere und reinere Ausbeute zu erzielen (Adsorption an Aluminiumoxyd, Extraktion nur in Butanol-Essigsäure) und die Verluste, die durch die nicht alles MBF erfassenden Lösungsmittel (Chloroform) und durch den nicht quantitativen Übergang des MBF in Komplexsalze, verursacht sind, $z u$ vermeiden und hierbei das MBF in die einzelnen Fraktionen zu verteilen, haben wir folgendes Verfahren ausgearbeitet:

\section{Eigene Isolierungsmethode des MBF aus dem Stuhl}

$20 \mathrm{~g}$ des frischen, ungetrockneten Stuhles, der nach vorheriger fleisch- und chlorophyllfreier Kost gesammelt wurde, werden erst mit Chloroform (Bilirubinbeseitigung), weiter mit schwacher Alkalilösung (Mesobilileukane-beseitigung) und dann nach Ansäuerung mit Petroläther (Sterkobilinogen- und Porphyrin-beseitigung) ausgewaschen. Aus der Chloroformauswaschung (nach Abdampfen) wird das Bilirubin nach Alkalisieren mit $\mathrm{NaOH}$ durch Bariumchloridlösung gefällt und aus dem Rest das MBF in ButanolEssigsäure eluiert. Aus dem Hauptmuster wird die Extraktion des MBF auch in Butanol-Essigsäure (10:1) dreimal unternommen. Die vereinigten Extrakte werden auf einige $\mathrm{ml}$ eingeengt und mit 
Methanol auf $150 \mathrm{ml}$ verdünnt. Die so vorbereitete Lösung nach der $\mathrm{NaOH}-\mathrm{Zugabe}$ zum Ausgleich der Ionenkonzentration auf $\mathrm{pH}=7$ wird mit pulverisiertem Aluminiumoxyd versetzt. Das verfärbte Aluminiumoxyd wird mit destilliertem Wasser und mit 1-proz. wässerigen $\mathrm{NaOH}-L$ ösung gewaschen. Die Auswaschungen enthalten kein MBF. Danach wird das MBF erneut mit ButanolEssigsäure eluiert und nach Verdampfen wird das Material in die einzelnen Lösungsmittel verteilt. Obzwar die quantitative Ausbeute des MBF durch die verschiedene Reihenfolge der einzelnen Auswaschungsmittel prinzipiell nicht beeinträchtigt wurde, empfehlen wir folgendes Verfahren: 1. Chloroform, 2. Amylalkohol, 3. Wasser, 4. Methanol, 5. Butanol und 6. Eisessig. Durch die Eluierung mit 5. und 6. werden außer allen vorhergehenden noch neue Fraktionen aufgefangen. Die einzelnen Fraktionen werden auf der Aluminiumoxydsäule durch Adsorptionschromatographie mit Flüssigkeiten, die zugleich Lösungsmittel der betreffenden Fraktion darstellen, gereinigt. Das aufgefangene MBF wird mit Butanol-Essigsäure (dem stärksten Lösungsmittel) gewaschen. Das gereinigte MBF wird in jeder Fraktion spektrophotometrisch bei $450 \mathrm{~m} \mu$ gemessen. Das zur Eichung notwendige MBF wurde nach der Methode von Sieder und MölLER dargestellt (7), nach der Trocknung gewogen und zur Herstellung der Eichkurve verwendet.

Bei der Bestimmung des MBF in der Galle ist das Vorgehen identisch; das Bilirubin der Galle wird hier durch Verdünnen mit Wasser und durch Alkalisieren mit $\mathrm{NaOH}$-Lösung polarisiert und durch Bariumchlorid gefällt (14).

\section{Isolierung des $\mathrm{MBF}$ aus dem Harn}

Eigene Erfahrungen, gestützt auf die Löslichkeit des MBF in polaren Lösungsmitteln, haben uns gelehrt, daß im Harn nur die weniger polymerisierten MBF vorliegen; dies ist auch physiologisch begreiflich, denn größere Makromoleküle können scheinbar weder das normale Nierenglomerulum passieren, noch durch die Tubuli ausgeschieden werden.

Bei der eigenen Arbeit konnten wir feststellen, daß die Adsorption des MBF am pulverisierten Aluminiumoxyd größere Ausbeute liefert als die Extraktion mit Butanol-Essigsäure. Die Reinheit des Adsorbats wat auch größer. Die von Gilbertsen und Mitarbeitern im Jahre 1959 benützte Methode zur Isolierung und quantitativen Bestimmung des MBF im Harn ist analog ihrer Methode für die Bestimmung des MBF im Stuhl, wie sie vorher beschrieben wurde.
Unsere eigene Isolierungstechnik für die "Bestimmung des MBF im Harn war die folgende:

Bei Gegenwart von Bilirubin und Bilirubinoiden im Urin werden dieselben mit Bariumchlorid und Petroläther beseitigt, wonach die Harnprobe (dreimal mit Wasser verdünnt) mit pulverisiertem Aluminiumoxyd bis zur vollständigen Farblosigkeit des Urins gesättigt wird. Das verfärbte Aluminiumoxyd verrät sich schon jetzt durch einen makroskopisch feststellbaren rostfarbenen Ton des sedimentierenden Aluminiumoxyds. Das Adsorbat wird dann mit Butanol-Essigsäure 10:1 eluiert; es genügt auch der bloße Eisessig zur totalen Bestimmung, weil die beiden Fraktionen des Urin-MBF (die Essigsäurefraktion und die Methanolfraktion) nicht nur in dem Eisessig löslich, sondern auch aus Aluminiumoxyd eluierbar sind.

Die Butanol-Eisessig-Lösung wird nach Verdampfen weiterer Reinigung unterworfen und zwar durch das Waschen in Chloroform und Petroläther. Nach erneutem Verdampfen wird das MBF teils im Methanol, teils in Eisessig gelöst. Die einzelnen Fraktionen werden dann durch die Adsorptionschromatographie gereinigt, wie es oben angeführt wurde. Nach Überführung in die entsprechenden Lösungsmittel wird die Messung bei $450 \mathrm{~m} \mu$ vorgenommen. Die qualitative Messung ergab ein Spektrum von kurvenförmiger Gestalt, mit dem Maximum bei $400-450 \mathrm{~m} \mu$, langsam zu höheren Wellenlängen abfallend; das Spektrum war mit dem von SIEDEL beschriebenen identisch. Der Stickstoffanteil betraf 8,6 bis $8,8 \%$ (Theorie: $8,8 \%$ ). Diese Methode ergab höhere Werte als die vorher angegebene.

Die Frage der Chromogene - Mesobilileukane und Bilileukane (11) - bedarf noch einer Erklärung. Durch das beschriebene Verfahren werden sowohl im Stuhl, als auch im Harn nur die Fuscine erfaßt, während die Chromogene außer Acht bleiben. Um auch die Fraktion der Chromogene zu erfassen, ist es ratsam, dieselben mit Isoamylalkohol (aus Harn) oder mit Alkalilösung (aus Stuhl) zu eluieren (8) und demnächst ihre Kondensation mit konzentrierter Salzsäure unter Erwärmung zu verwirklichen. Die von uns angeführten Bestimmungsmethoden des MBF im Stuhl, in der Galle und im Harn sind wohl anspruchsvoll, aber sie ermöglichen eine verläßliche Feststellung der Anwesenheit und Menge des MBF. Für die Bedürfnisse der normalen und pathologischen Zustände des Austausches der Pyrrolfarbstoffe ist die Empfindlichkeit dieser Methoden vollauf genügend.

Für die Mitarbeit und materielle Unterstützung sage ich Herrn Professor Dr. M. Netoušek und dem Herrn Primarius Dr. O. SoušEK, für den chemischen Rat RNDr. B. TVAROHA großen Dank.

\section{Literatur}

1. André, R., B. Dreyfus und C. Sultan, Nouv. Rev. Franc. Haem. 3, 189 (1963). - 2. Gilbertsen, A. S., P. T. LowrY, V. H. Hawkinson und C. J. Watson, J. Clin. Invest. 7, 1166 (1959). - 3. Gilbertsen, A. S., V. H. Hawkinson und C. J. Watson, J. Clin. Invest. 7, 1175 (1959). - 4. Moravec, M. und M. NetoušEK, Blut 9, 182 (1963). - 5. NetoušEK, M., Acta med. Acad. Sci. hung. 9, 143 (1956). - 6. Srrck, W., Münch. med. Wschr. 92, 1275 (1950). - 7. SIEDEL, W. und H. Möller, HoppeSeyler's Z. physiol. Chem. 259, 113 (1939). - 8. Sreder, W., in Hoppe-Seyler-Thierfelder, Handbuch der physiol. und pathol. chemischen Analyse II. B., Springer-Verlag, Berlin (1950). -
9. Huggrns, M. L., Physical Chemistry of High Polymers, Wiley, New York (1958). - 10. MEYER, K. H. und H. MARK, Makromolekulare Chemie, Geest \& Portig, Leipzig (1950). - 11. Sieder, W., W. PöLnitz und F. EisenReich, Naturwissenschaften 34, 314 (1947). - 12. Sieder, W., W. Stich und F. Eisenreich, Naturwissenschaften 35, 316 (1948). - 13. Watson, C. J., A. S. GiLBERTSEN, P. T. LOWRY und V. H. HAWKINSON, VII. Internat. Soc. Haem. Rom 2. V., 1 (1958). - 14. Israels L. G., J. Skadenberg, H. GuYdA, W. ZingG und A. Zipursky, Brit. J. Haem. 9, 50 (1963). 